\title{
Disentangling the relation between intentions, planning, and behaviour: A moderated mediation analysis
}

\author{
A. U. Wiedemann ${ }^{a *}$, B. Schüz ${ }^{b}$, F. Sniehotta ${ }^{c}$, \\ U. Scholz ${ }^{d}$ and R. Schwarzer ${ }^{a}$ \\ ${ }^{a}$ Freie Universität Berlin, Department of Health Psychology, Habelschwerdter Allee 45, 14195, \\ Berlin, Germany; ${ }^{b}$ Deutsches Zentrum für Altersfragen (German Centre of Gerontology), \\ Manfred-von-Richthofen-Straße 2, 12101, Berlin, Germany; ${ }^{c}$ University of Aberdeen, Aberdeen, \\ AB24 2UB, United Kingdom; ${ }^{d}$ University of Zurich, Social and Health Psychology, 8050, Zürich \\ Switzerland
}

(Received 12 July 2007; final version received 28 January 2008)

\begin{abstract}
Action planning is assumed to mediate between intentions and health behaviours. Moreover, intentions are assumed to moderate the planning-behaviour relation, because people with high intentions are more likely to enact their plans. The present studies extend these suppositions by integrating both assumptions to a novel and parsimonious model of moderated mediation: the mediation effect is hypothesised to be stronger in individuals who report higher intention levels. In two longitudinal studies on physical activity $(N=124)$ and interdental hygiene $(N=209)$, intentions and action planning were assessed at baseline, and behaviour was measured four (Study 1), and respectively, three (Study 2) months later. The moderated mediation hypothesis was tested with continuously measured intentions using regression analyses with non-parametric bootstrapping. Results from both studies suggest that levels of intentions moderate the mediation process: The strength of the mediated effect increased along with levels of intentions. Planning mediates the intention-behaviour relation, if individuals hold sufficient levels of intentions. Implications for theory advancement and intervention development are discussed.
\end{abstract}

Keywords: intentions; action planning; health behaviour change; moderated mediation; physical activity; dental hygiene

\section{Introduction}

\section{Determinants of health behaviour}

Health behaviour theories such as the Theory of Planned Behaviour (Ajzen, 1991) or the Protection Motivation Theory (Maddux \& Rogers, 1983) propose intentions to be proximal determinants of behaviour. However, people often fail to act on their intentions (Sheeran, 2002). To understand why some people succeed in translating their intentions into behaviour whereas others do not, individual differences in post-intentional processes of goal pursuit such as action planning should be considered (Abraham, Sheeran, \& Johnston, 1998).

\footnotetext{
*Corresponding author. Email: amelie.wiedemann@fu-berlin.de
} 


\section{Intentions, action planning, and behaviour}

Action planning is a prospective self-regulatory strategy that helps to translate intentions into action. Thus, it serves the purpose of superordinate goal intentions such as 'I intend to be more physically active'. While intentions describe a desired goal state, action planning refers to mental simulations of linking concrete behavioural responses to situational cues in order to achieve this goal state. These simulations create cognitive representations of the target situation that make situational cues easily detectable and, thus, the behavioural response more likely as soon as the critical situation occurs (Gollwitzer, 1999). A conceptual distinction can be made between experimentally induced if-then planning (implementation intentions; Gollwitzer, 1999) as opposed to self-reported action planning. The latter refers to responses to psychometric scales asking people whether they have formed action plans or not (e.g. Sniehotta, Scholz, \& Schwarzer, 2005), and predicts behaviour over and above goal intentions up to 12 months (Ziegelmann, Luszczynska, Lippke, \& Schwarzer, 2007). The present studies investigate the interplay between these self-induced planning processes, intentions and behaviour.

\section{Interrelation between intentions, planning, and behaviour: mediation and moderation}

It has been hypothesised that holding intentions instigates individuals to engage in action planning, which in turn affects the active behaviour-changing process (Schwarzer et al., 2007). Indeed, several studies found action planning to partially mediate the intentionbehaviour relation and to add explained variance to the prediction of health behaviours (e.g. Norman \& Conner, 2005, Study 2; Scholz, Schüz, Ziegelmann, Lippke, \& Schwarzer, in press; Schwarzer et al., 2007, Study 1-3; full mediation, Sniehotta et al., 2005). However, the evidence is inconclusive as some studies failed to find mediation effects of planning (Norman \& Conner, 2005, Study 1; Schwarzer et al., 2007, Study 4; White, Terry, \& Hogg, 1994).

This suggests that the complex relations between intentions, planning and behaviour cannot be subsumed within simple mediation models, but might also depend on moderating variables. Thus, mediation mechanisms might differ in subgroups of participants (e.g. sex, age groups). For example, the degree to which planning mediates between intentions and behaviour has been found to be higher in older than in younger individuals (Reuter, Ziegelmann, Wiedemann, Lippke, \& Schüz, 2007). Statistically speaking, this represents a case of moderated mediation (Edwards \& Lambert, 2007; Preacher, Rucker, \& Hayes, 2007): The amount to which the mediator (Me) translates the effect of the independent variable (IV) on the dependent variable (DV) may depend on the levels of a moderator variable (Mo).

One potential moderator for the degree to which planning mediates the intentionbehaviour relationship is intention itself. Intentions moderate the planning-behaviour relation, because (a) high intentions increase the encoding depth of the situational cue and the cue-response link, and, thus, the efficacy of if-then plans; and (b) intention strength influences the degree of specification of if-then plans, which in turn predicts the likelihood of behaviour enactment (see Sheeran, Milne, Webb, \& Gollwitzer, 2005, for a review). The fact that in individuals with high intentions planning is more closely related to behaviour (moderation) might also impact on the mediation process: Whether intentions that affect behaviour via planning (mediation) might depend on intention levels. 


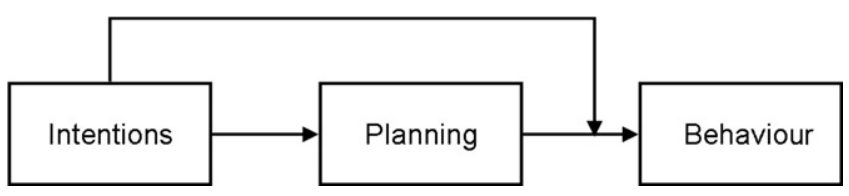

Figure 1. Conceptual moderated mediation model.

Therefore, the aim of the present studies is to analyse whether planning (Me) mediates the effect of intentions (IV) on behaviour (DV) as a function of the underlying level of intentions (Mo). Of course, models integrating other moderators (e.g. intention stability; Conner \& Godin, 2007) or additional mediators (e.g. phase-specific self-efficacy; Scholz, et al., 2005) are plausible as well. However, the present studies aimed at testing whether the independent variable additionally serves as a continuous moderator variable, thus resulting in a parsimonious model of the intention-planning-behaviour relation. This model satisfies one major principle of theory building, the principle of parsimony (Preacher et al., 2007). Components of this model (simple mediation and moderation) have been tested before, but are now combined to a model of moderated mediation. To our knowledge, the present study is the first one to test moderated mediation for this research question.

\section{Aim and hypothesis}

The present studies aim at

(i) Testing a parsimonious model of moderated mediation (Figure 1), which integrates two assumptions in one model: (a) Intentions influence behaviour through action planning processes (mediation), and (b) the strength of the planning-behaviour association depends on the level of intentions (moderation). In particular, we hypothesised that mediation by action planning is particularly strong in people with high intentions to engage in health behaviour (moderated mediation). As a preliminary analysis, the indirect effect of intentions on behaviour via action planning (simple mediation) is tested.

(ii) Exemplarily presenting a systematic method of testing this special model of moderated mediation as suggested and instigated by Preacher et al. (Model 1; 2007), with rationale and methods being applicable to other models of moderated mediation as well.

\section{Two studies on physical activity and interdental hygiene}

Moderated mediation is examined in two samples dealing with two health behaviours. Study 1 assessed physical activity in the context of cardiac rehabilitation. Despite high motivation to change their lifestyle after discharge from inpatient treatment (e.g. Sniehotta, Schwarzer, Scholz, \& Schüz, 2005), the majority of people with coronary heart disease fail to follow the recommendations for physical activity in the long run (Willich et al., 2001). For the implementation of a physically active lifestyle, action planning proved beneficial: Action planning mediated the intention-behaviour relation, and effects of action planning were moderated by intentions (Norman \& Conner, 2005; Scholz et al., in press). Study 2 focused on interdental hygiene. Interdental hygiene is 
effective for the prevention of oral diseases such as caries and periodontitis (Bellamy et al., 2004), yet few people adhere to daily interdental hygiene as recommended by the American Dental Association (ADA, 2005; Bader, 1998). In a longitudinal study on social-cognitive variables, action planning emerged as the only predictor of adherence to dental flossing recommendations when controlling for intentions (Schüz, Sniehotta, Wiedemann, \& Seemann, 2006). Intentions did not moderate the effect of planning on flossing. Thus, in contrast to research on physical activity, there is little evidence that intentions moderate the influence of planning on interdental hygiene.

\section{Method}

\section{Analytical procedure}

\section{Preliminary analysis: simple mediation}

The product-of-coefficients strategy with bootstrapping was used to test strength and significance of the indirect effect. Bootstrapping is a non-parametric method for assessing indirect effects (Preacher \& Hayes, 2004; Preacher et al., 2007). Exact normal distribution may only be found in large samples, but bootstrapping overcomes several problems with non-normally distributed variables such as power problems. Thus, the indirect effect was estimated by first regressing planning (Me) on intentions (IV), and subsequently regressing behaviour (DV) on planning (Me) and intentions (IV). The indirect effect was then quantified as the product of the mean bootstrapped sample estimates of the regression coefficients ('Me on IV' ' $\mathrm{DV}$ on ME controlling for IV'). The standard deviation of the estimate of the indirect effect obtained over 5000 bootstrapped resamples is the estimated standard error of the mean indirect effect (Preacher \& Hayes, 2004). Based on this information, bootstrap confidence intervals were generated for the indirect effect.

\section{Hypothesis on moderated mediation}

In order to test whether the indirect effect depends on intentions levels (Mo), coefficients were estimated independently in two regression analyses using bootstrapping. First, action planning (Me) was regressed on intentions (IV). Subsequently, behaviour (DV) was regressed on intentions (IV), planning (Me), and the interaction between intentions and planning (Mo* Me; using mean centered variables). An overall effect of the IV on the Me is a necessary precondition for moderated mediation: A significant interaction effect $(\mathrm{Mo} * \mathrm{Me})$ on behaviour is only indicative of moderated mediation if intentions (IV) also affect action planning.

Given a significant interaction effect, regression analyses were then conducted on several values of the moderator to obtain the degree to which mediation varies depending on the level of the moderator. Variables were z-standardised, and indirect effects are reported at $M$ and $\pm 1 \mathrm{SD}$ of the moderator. Bias-corrected bootstrapping was applied as it produces more accurate confidence intervals (MacKinnon, Lockwood, \& Williams, 2004). Additionally, an extension of the Johnson-Neyman technique to moderated mediation was applied (Preacher et al., 2007). This technique tests the significance of the indirect effect on a large range of values of the moderator until the value of the moderator is identified for which the conditional indirect effect is just statistically significant at a set $\alpha$-level (here, $\alpha=0.05$ ). Values of the moderator for which the mediation effect is significant constitute the region of significance of the indirect effect. 
Table 1. Sample characteristics.

\begin{tabular}{lcc}
\hline & Study 1: Physical activity & Study 2: Interdental hygiene \\
\hline Participation: N (\% of baseline) & $124(72.5 \%)$ & $209(62.9 \%)$ \\
Age in years: $M$ (SD; Range) & $60.3(10.4 ; 33-84)$ & $45.1(16.8 ; 18-81)$ \\
Gender: female & $23(18.3 \%)$ & $139(66.5 \%)$ \\
Marital status: married & $98(77.8 \%)$ & $134(64.1 \%)$ \\
Education: higher education & $24(19.1 \%)$ & $108(51.7 \%)$ \\
Employment: working for pay & $66(52.4 \%)$ & $108(51.7 \%)$ \\
\hline
\end{tabular}

\section{Study 1: Physical activity}

\section{Participants and procedure}

Persons with medical indication to adhere to regular physical activity were recruited for Time 1 (T1) assessments during their second week of stay in one of three cardiac rehabilitation centers. After giving informed consent, 171 participants completed T1 questionnaires assessing intentions, action planning and sociodemographic variables. The four-month follow-up questionnaires (T2) assessed physical activity and were sent to the participants with a prepaid return envelope. Participation was voluntary and unpaid in both Study 1 and Study 2. Both studies were conducted in accordance with the WMA declaration of Helsinki (WMA, 2004).

The longitudinal sample comprised 124 participants $(72.5 \%$ of baseline), $81.7 \%$ of them were men (Table 1). Mean age was 60.3 years $(\mathrm{SD}=10.4$; range 33 to 84 years). Drop-out analyses indicated that participants who continued study participation reported significantly higher baseline intentions than those who did not $(F(1,169)=9.77, p<0.05)$, but did not differ with regard to planning, physical activity and sociodemographic variables (age, sex, as well as marital, educational and occupational status, all $p \mathrm{~s}>0.05$ ). Missing data ( $<10 \%$ on all variables in both studies) were imputed using the Expectation Maximization (EM) algorithm (Enders, 2001) in SPSS 13.

\section{Measures}

Item examples of both studies given below are translations from German. Unless otherwise stated, response formats were four-point Likert scales, ranging from completely disagree (1) to completely agree (4). Scale scores were obtained by averaging item responses.

Behavioural intentions were measured by six items (Sniehotta et al., 2005), such as 'I intend to be physically active several times per week'. Means, standard deviations, reliability coefficients and intercorrelations of all variables are displayed in Table 2.

Action planning was assessed with four items: 'I have made a detailed plan on [when; where; how; how often] to exercise' (Sniehotta et al., 2005).

Physical activity was assessed by an adaptation of the International Physical Activity Questionnaire (Booth, 2000) to the special characteristics of the cardiac sample. Participants indicated how often per week and how long per session they performed (a) vigorous exercise (e.g. swimming), (b) fitness activities (e.g. gymnastics), (c) game sports (e.g. volleyball), (d) moderate exercises to train muscle strength and (e) other prescribed exercises on average during the last four weeks. Total physical activity was the sum of the multiplied exercise sessions per week by minutes per session. All measures were significantly associated with each other (Table 2). 
Table 2. Descriptive statistics, reliability and intercorrelations for time 1 intentions, T1 planning and time 2 behaviour.

\begin{tabular}{|c|c|c|c|c|c|c|}
\hline & \multicolumn{3}{|c|}{ Study 1: Physical activity } & \multicolumn{3}{|c|}{ Study 2: Interdental hygiene } \\
\hline & Intention & Planning & Behaviour & Intention & Planning & Behaviour \\
\hline$\alpha$ & 0.84 & 0.93 & - & 0.64 & 0.90 & - \\
\hline$M$ & 3.41 & 3.06 & 123.7 & 3.41 & 3.42 & 18.36 \\
\hline SD & 0.55 & 1.01 & 131.19 & 0.68 & 0.72 & 11.13 \\
\hline Intention & - & $0.66 * *$ & $0.36 * *$ & - & $0.51 * *$ & $0.37 * *$ \\
\hline Planning & - & - & $0.36^{* *}$ & - & - & $0.47 * *$ \\
\hline
\end{tabular}

Note: $* * p<0.01$.

\section{Results}

\section{Mediation}

Results from bootstrapping yielded a significant mean indirect effect of intentions on physical activity through action planning of $\beta=0.15(\mathrm{SE}=0.06)$ with a $95 \%$ confidence interval from 0.05 to 0.19 . Planning only partially mediated the intention-behaviour relation, as intentions still had a significant direct effect on behaviour $(\beta=0.23, p<0.05)$, albeit lower than without planning being controlled for $(\beta=0.38, p<0.01)$.

\section{Moderated mediation}

The moderated mediation hypothesis was tested by two regression analyses. First, planning (Me) was predicted by intentions (IV; $\beta=0.68, p<0.01$ ). Subsequently, physical activity was predicted by intentions $(\beta=0.32, p<0.01)$, action planning $(\beta=0.22$, $p<0.05)$, and the intention $*$ planning interaction $(\mathrm{Mo} * \mathrm{Me} ; \beta=0.18, p<0.05)$, with $19 \%$ of the behavioural variance being explained. The significant interaction effect supported the assumption of moderated mediation (Figure 2). ${ }^{1}$

Given the overall significant interaction term, significance tests were conducted on the hypothesis that the conditional indirect effect equals zero at specific values $(M, \pm 1 \mathrm{SD})$ of the moderator. Action planning mediated the effect of intentions on physical activity at the mean and at high levels ( $+1 \mathrm{SD})$ of intentions, but not when intentions were low $(-1 \mathrm{SD}$; see Table 3). In particular, the strength of the conditional indirect effect increased along with levels of intentions $(\beta=0.15$ at $M$, and $\beta=0.28$ at $+1 \mathrm{SD}$; both $p$ s $<0.01)$.

The region of significance had its lower bound at intentions $=-0.22$ (z-value; $p<0.05$ ) and its upper bound at 1.17 ( $z$-value; $p<0.01)$. The lower bound describes the lowest $z$-value of the moderator, for which the indirect effect is significant. Here, the upper bound equals the largest value of the moderator within the data range, as the mediation effect could not be tested at higher values of the moderator.

In Figure 3 the indirect effect is plotted at all values of the moderator with a $95 \%$-confidence band. The horizontal line denotes indirect effects of zero. The vertical line represents the lower boundary of the region of significance. The indirect effect of intentions on physical activity via planning is significant (region of significance) where the confidence band does not contain zero. In line with the assumptions, the indirect effect increases along with intention levels. 


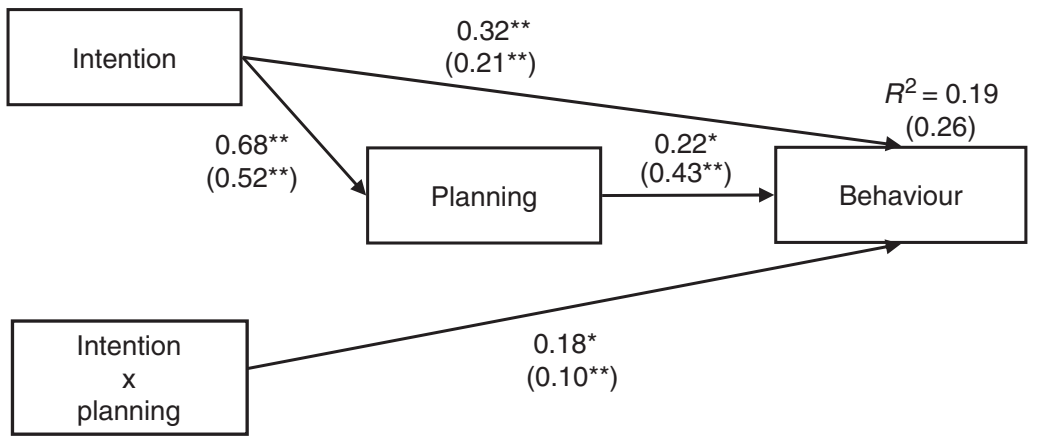

Note: ${ }^{* *} p<0.01 ;{ }^{*} p<0.05$

Figure 2. Results of regression analyses for moderated mediation of Study 1 and Study 2 (in parentheses).

Table 3. Bootstrapped indirect effects of intentions on behaviour via planning at specific values of the moderator (intentions).

\begin{tabular}{lccccccccc}
\hline & \multicolumn{3}{c}{ Study 1: Physical activity } & & \multicolumn{3}{c}{ Study 2: Interdental hygiene } \\
\cline { 2 - 3 } Intention & $\beta$ & SE & LL BCA & UL BCA & & & SE & LL BCA & UL BCA \\
\hline -1 SD & 0.02 & 0.07 & -0.12 & 0.16 & & $0.16^{* *}$ & 0.04 & 0.06 & 0.23 \\
Mean & $0.15^{* *}$ & 0.06 & 0.04 & 0.27 & & $0.20^{* *}$ & 0.05 & 0.11 & 0.31 \\
+1 SD & $0.28^{* *}$ & 0.09 & 0.10 & 0.46 & & $0.25^{* *}$ & 0.08 & 0.12 & 0.43 \\
\hline
\end{tabular}

Note: $* * p<0.01 ; N=5000$ Bootstrapping resamples; LL BCA and UL BCA $=$ Lower level and Upper level of the bias corrected and accelerated confidence interval for $\alpha=0.05$.

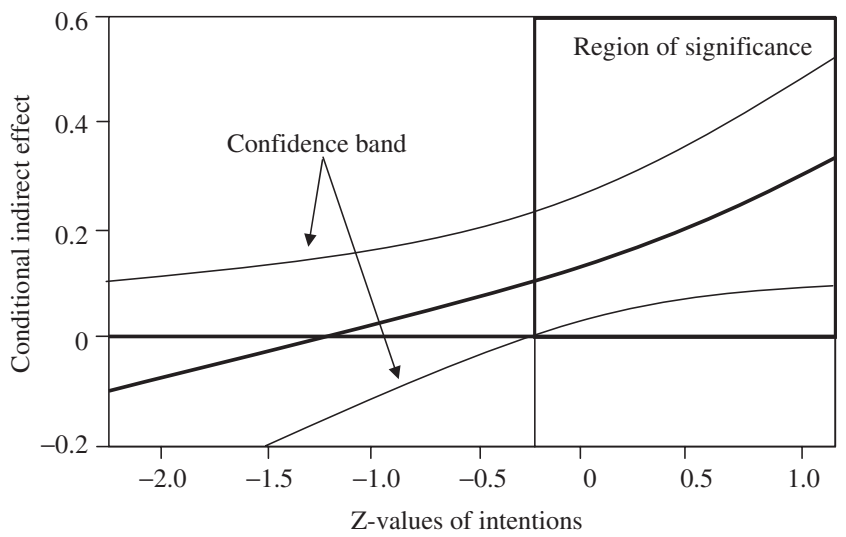

Figure 3. Study 1: Moderated indirect effect of intentions on physical activity through planning with a $95 \%$-confidence band.

\section{Study 2: Interdental hygiene}

\section{Participants and procedure}

Participants were recruited in dental practices in Germany. Exclusion criteria were being younger than 18 years (full age) and having full dental prosthetics. A total of 332 persons gave informed consent and received Time 1 (T1) questionnaires including scales on 
intentions, action planning and sociodemographic variables. Additionally, participants were provided with dental floss or interdental brushes as well as interdental hygiene instructions. Time 2 (T2) questionnaires assessed interdental hygiene behaviour and were mailed three months after T1 along with prepaid return envelopes.

Longitudinal data are available from 209 participants $(62.9 \%$ of baseline); $66.5 \%$ of them were women. Mean age was 45.1 years $(\mathrm{SD}=16.8$; range 18 to 81 years). Drop-out analyses revealed higher intentions $(F(1,330)=4.42, p<0.05)$ and higher levels of action planning $(F(1,330)=11.15, p<0.01)$ in the longitudinal sample. No differences in interdental hygiene and sociodemographic variables (age, sex and marital, educational and occupational status, all $p s>0.05$ ) were found.

\section{Measures}

Intentions were assessed using three items based on Rise, Åstrøm, and Sutton (1998). The stem 'I intend to...' was followed by items such as '... clean my interdental spaces daily.'

Action planning was measured by five items: 'I have made a detailed plan on [when; how; how often; how long; combined with which regular behaviour] to clean my interdental spaces' (Schüz et al., 2006).

Interdental hygiene was measured using an open-ended single item: 'How often did you clean your interdental spaces during the last month?' Self-report measures of this kind proved valid against objective measures of flossing behaviour (correlations between flossing self-reports and residual floss ranged between 0.59 and 0.80; McCaul, Glasgow, \& Gustafson, 1985; Schüz et al., 2006; Sniehotta, Araújo-Soares, \& Dombrowski, 2007). Descriptive data are displayed in Table 2.

\section{Results}

\section{Mediation}

Results from bootstrapping yielded a mean indirect effect of $\beta=0.18 \quad(\mathrm{SE}=0.04)$ with a $95 \%$-confidence interval from 0.11 to 0.27 . However, planning was only a partial mediator, as intentions still had a significant direct effect on behaviour $(\beta=0.18, p<0.01)$, albeit lower than without planning being controlled for $(\beta=0.36$, $p<0.01)$.

\section{Moderated mediation}

As in Study 1, intentions (IV) predicted action planning (Me; $\beta=0.52, p<0.01$ ) and intentions $(\beta=0.21, p<0.01)$; action planning $(\beta=0.43, p<0.01)$ and the intention $*$ planning interaction $(\mathrm{Mo} * \mathrm{Me} ; \beta=0.10, p<0.01)$ predicted interdental hygiene (Figure 2). The significant interaction supported the moderated mediation hypothesis.

Bootstrapping analyses indicated a significant indirect effect of intentions on behaviour on all three chosen values of intentions (see Table 3), which increased along with levels of the moderator $(\beta=0.16$ at $-1 \mathrm{SD}, \beta=0.20$ at $M$, and $\beta=0.25$ at $+1 \mathrm{SD}$; all $p \mathrm{~s}<0.01)$.

The region of significance had its lower bound at $z=-1.86(p<0.05)$ and its upper bound at $z=1.22(p<0.01)$ of the moderator. The upper bound equals the largest value of the moderator within the data range in Study 2 as well, as the indirect effect could not be tested at a higher value of the moderator. Figure 4 displays results for Study 2 


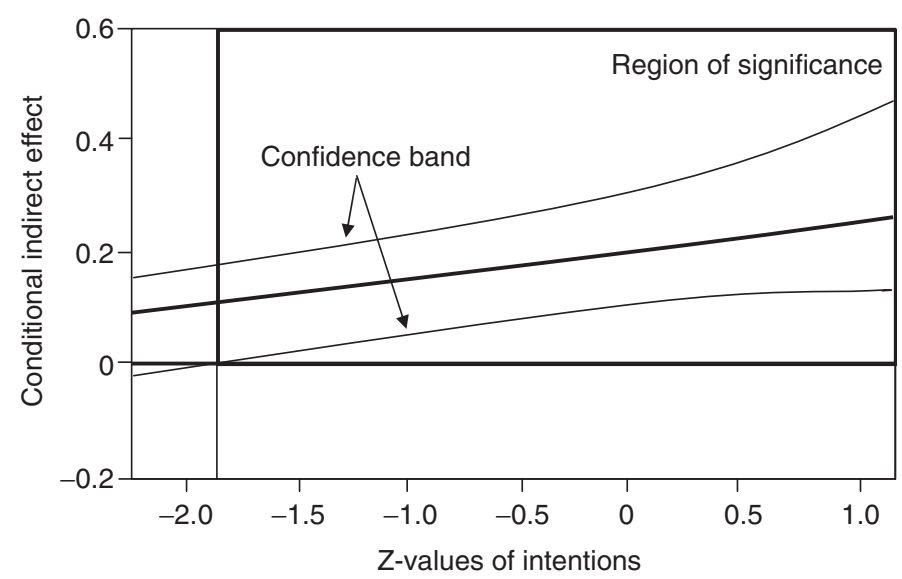

Figure 4. Study 2: Moderated indirect effect of intentions on interdental hygiene through planning with a $95 \%$-confidence band.

analogous to Figure 3 for Study 1. As hypothesised, the indirect effect increases along with intention levels.

\section{Discussion}

This article analysed in two studies whether the mediation effect of the intention-behaviour relation by planning varies due to different levels of intentions, thus providing first evidence for this parsimonious model of moderated mediation.

\section{Interrelations between intentions, planning, and health behaviour}

The present findings emphasise the importance of both intentions and planning for behaviour change, as both had main effects on behaviour (Figure 2). However, they work best in orchestration: intentions are translated into action by planning (mediation), and the effects of planning on behaviour are moderated by intentions, indicating that people with high intentions are more likely to enact their plans (moderation). This is in line with assumptions of stage theories and recent experimental research (cf. Lippke, Ziegelmann, \& Schwarzer, 2004; Sheeran, Webb, \& Gollwitzer, 2005).

As an extension of previous research, we examined whether this moderation effect influences the translation of intentions into action by planning. In both studies, the strength of the partial mediation effect increased along with levels of intentions (Table 3, Figures 3 and 4): Planning helps to translate intentions into behaviour particularly well in individuals with moderate to high intentions, as they are more likely to act on their plans (Sheeran, Milne, Webb, \& Gollwitzer, 2005). Hence, this refinement might explain the inconsistent results of previous studies regarding the role of action planning as a mediator between intentions and behaviour (e.g. Norman \& Conner, 2005; Schwarzer et al., 2007).

The indirect effect represents a facilitating mechanism, i.e. people with high intentions make more use of planning and, thus, are more likely to act (Sutton, 2008). However, by taking moderated mediation into account, we acknowledge that this automatic process works differently in subgroups of individuals. Thus, moderated mediation allows a better 
description of the causal mechanisms by which intentions influence behaviour: intentions affect behaviour via three pathways, (a) directly, as indicated by a main effect on behaviour, and indirectly via (b) the mediation path and (c) via the moderator effect which catalyzes the mediation effect.

\section{Implications}

The present findings have several implications. Theory building might gain from consideration of such moderated mediation assumptions, because they help to explain how indirect effects vary at different levels of moderating conditions. Theoretical considerations may also be translated into theory-based interventions: Interventions based on the simple mediation model would promote intentions which would lead to higher planning and, thus, to higher performance. However, if the effects of such interventions would be estimated by simple mediation models, the actual effect of intentions on the behavioural outcome would be underestimated: direct effects as well as the moderating effects of intentions would be disregarded. In addition, to 'jump into the assumed causal chain' (Sutton, 2008) by promoting planning directly should be less effective than a combined intervention on intentions and planning, given the moderating effect of intentions.

Thus, interventions might work best if they target intentions as precondition or in combination with planning, because intentions (as IV) not only promote planning processes but also enhance the likelihood that plans will be enacted (as Mo). Thus, even small changes in intention to act might affect whether planning interventions are beneficial for behaviour change, as our results indicate that indirect effects through planning develop only above a certain threshold of intentions and gradually increase with small increments of intentions (Mo).

However, the total magnitude of intentions necessary for planning to mediate between intentions and behaviour is assumed to differ between simple and complex behaviours, as intentions had to exceed a higher threshold in physical activity than in interdental hygiene. This was indicated by the lower boundary of the region of significance for interdental hygiene.

The present findings are based on non-experimental longitudinal data. Thus, suggestions for interventions are tentative. Future experimental studies should compare the effect variance explained by direct, indirect and moderator effects of intentions and explicitly test the differential effectiveness of simple planning and planning plus goal setting interventions.

\section{Limitations}

Limitations of the present studies include that there was systematic dropout, as indicated by baseline differences in levels of intentions (Study 1 and 2) and action planning (Study 2) between dropouts and participants remaining in the study. Therefore, the present findings should be interpreted cautiously. A second limitation, the high attrition rate in both studies, might be due to voluntary participation without compensation. Third, although evidence exists that self-reports of physical activity and interdental hygiene are valid (e.g. Miller, Freedson, \& Kline, 1994; Schüz et al., 2006; Sniehotta et al., 2005), objective measures of the dependent variables are desirable. Fourth, the cross-sectional measurement of intentions and planning does not allow strong inferences for cause-and-effect relations. Yet, by having predictor and mediator assessed at the same measurement point 
in time, confounds with temporal proximity between mediator and criterion are circumvented. Fifth, including baseline behaviour might have added variance explained to future behaviour, but might also have masked the theoretically more interesting effects of social-cognitive variables without providing further insights into the processes leading to behaviour (e.g. Ajzen, 2002).

\section{Conclusions and outlook}

The present studies tested a specific model in which intentions serve as independent variable of a simple mediation process and additionally as moderating variable. The findings provide evidence that mediation effects are not constant across different levels of intentions and indicate that more research is needed beyond the mere investigation of planning as a mediator by taking possible interaction effects into account.

Additionally, the intention-behaviour relation was only partially mediated by planning in both studies. Future research might therefore test the existence of additional mediators such as action control (Sniehotta, Scholz et al., 2005) or maintenance and recovery self-efficacy (Scholz et al., 2005) in multiple mediation models.

Moderators can influence the mediation process in several ways. More research is needed that examines whether the mediation between intentions and behaviour by action planning is influenced by further moderators of the intention-planning relation (IV-Me), the planning-behaviour relation as in the present studies (Me-DV), or both relations. Extensive research by Conner and Godin (2007) indicated that intention stability moderates the intention-behaviour relation across several types of health behaviours, samples and time intervals. Future research might examine whether intention stability also moderates the intention-planning or the planning-behaviour relation.

Since different levels of intentions were necessary for planning to mediate the intention-behaviour relation, further research might also focus on thresholds of moderators that determine the occurrence of causal relations. For instance, research by Priester and Petty (1996) provides correlational and experimental evidence for the usefulness of a gradual threshold model to describe the influence of attitudinal reactions on subjective ambivalence. This might guide future research on the relation between intentions, planning and behaviour.

\section{Note}

1. Analyses using a normal-theory approach yielded similar results. Only results from bootstrapping are reported here.

\section{Acknowledgement}

The first author was supported by a stipend from the Konsul Karl and Dr. Gabrielle Sandmann Foundation. Study 2 was supported by Grant Nr 1-110804 from GABA International AG to Falko F. Sniehotta and Benjamin Schüz.

\section{References}

Abraham, C., Sheeran, P., \& Johnston, M. (1998). From health beliefs to self-regulation: Theoretical advances in the psychology of action control. Psychology and Health, 13, 569-591. 
ADA. (2005). Cleaning your teeth and gums (Oral hygiene). Retrieved May 11, 2007, from http://www.ada.org/public/topics/cleaning_faq.asp\#4

Ajzen, I. (1991). The theory of planned behavior. Organizational Behavior and Human Decision Processes, 50, 179-211.

Ajzen, I. (2002). Residual effects of past on later behavior: Habituation and reasoned action perspectives. Personality and Social Psychology Review, 6, 107-122.

Bader, H.I. (1998). Floss or die: Implications for dental professionals. Dentistry Today, 17, 76-78, $80-82$.

Bellamy, P., Barlow, A., Puri, G., Wright, K.I., Mussett, A., \& Zhou, X. (2004). A new in vivo interdental sampling method comparing a daily flossing regime versus a manual brush control. Journal of Clinical Dentistry, 15, 59-65.

Booth, M.L. (2000). Assessment of physical activity: An international perspective. Research Quarterly for Exercise and Sport, 71, 114-120.

Conner, M., \& Godin, G. (2007). Temporal stability of behavioural intention as a moderator of intention-health behaviour relationships. Psychology and Health, 22, 875-897.

Edwards, J.R., \& Lambert, L.S. (2007). Methods for integrating moderation and mediation: A general analytical framework using moderated path analysis. Psychological Methods, 12, 1-22.

Enders, C.K. (2001). A primer on maximum likelihood algorithms available for use with missing data. Structural Equation Modeling, 8, 128-141.

Gollwitzer, P.M. (1999). Implementation intentions: Strong effects on simple plans. American Psychologist, 54, 493-503.

Lippke, S., Ziegelmann, J.P., \& Schwarzer, R. (2004). Initiation and maintenance of physical exercise: Stage-specific effects of a planning intervention. Research in Sports Medicine, 12, $221-240$.

MacKinnon, D.P., Lockwood, C.M., \& Williams, J. (2004). Confidence limits for the indirect effect: Distribution of the product and resampling methods. Multivariate Behavioral Research, 39, 99-128.

Maddux, J.E., \& Rogers, R.W. (1983). Protection motivation and self-efficacy: A revised theory of fear appeals and attitude change. Journal of Experimental Social Psychology, 19, 469-479.

McCaul, K.D., Glasgow, R.E., \& Gustafson, C. (1985). Predicting levels of preventive dental behaviors. Journal of the American Dental Association, 111, 601-605.

Miller, D.J., Freedson, P.S., \& Kline, G.M. (1994). Comparison of activity levels using the Caltrac accelerometer and five questionnaires. Medicine and Science in Sports and Exercise, 26, 376-382.

Norman, P., \& Conner, M. (2005). The theory of planned behavior and exercise: Evidence for the mediating and moderating roles of planning on intention-behavior relationships. Journal of Sport \& Exercise Psychology, 27, 488-504.

Preacher, K.J., \& Hayes, A.F. (2004). SPSS and SAS procedures for estimating indirect effects in simple mediation models. Behavior Research Methods, Instruments, \& Computers, 36, 717-731.

Preacher, K.J., Rucker, D.D., \& Hayes, A.F. (2007). Addressing moderated mediation hypotheses: Theory, methods, and prescriptions. Multivariate Behavioral Research, 42, 185-227.

Priester, J.R., \& Petty, R.E. (1996). The gradual threshold model of ambivalence: Relating the positive and negative bases of attitudes to subjective ambivalence. Journal of Personality and Social Psychology, 71, 431-449.

Reuter, T., Ziegelmann, J.P., Wiedemann. A.U., Lippke, S., \& Schüz, B. (2007). Beyond good resolutions: Planning mediates the intention-behavior relation as a function of age. Manuscript submitted for publication.

Rise, J., Åstrøm, A.N., \& Sutton, S. (1998). Predicting intentions and use of dental floss among adolescents: An application of the theory of planned behavior. Psychology and Health, 13, 223-236. 
Scholz, U., Schüz, B., Ziegelmann, J.P., Lippke, S., \& Schwarzer, R. Beyond behavioural intentions: Planning mediates between intentions and physical activity. British Journal of Health Psychology (in press).

Scholz, U., Sniehotta, F.F., \& Schwarzer, R. (2005). Predicting physical exercise in cardiac rehabilitation: The role of phase-specific self-efficacy beliefs. Journal of Sport and Exercise Psychology, 27, 135-151.

Schüz, B., Sniehotta, F.F., Wiedemann, A., \& Seemann, R. (2006). Adherence to a daily flossing regimen in university students: Effects of planning when, where, how, and what to do in the face of barriers. Journal of Clinical Periodontology, 33, 612-619.

Schwarzer, R., Schüz, B., Ziegelmann, J.P., Lippke, S., Luszczynska, A., \& Scholz, U. (2007). Adoption and maintenance of four health behaviors: Theory-guided longitudinal studies on dental flossing, seat belt use, dietary behavior, and physical activity. Annals of Behavioral Medicine, 33, 156-166.

Sheeran, P. (2002). Intention-behaviour relations: A conceptual and empirical review. In M. Hewstone \& W. Stroebe (Eds.), European review of social psychology (Vol. 12, pp. 1-36). Chichester, UK: John Wiley \& Sons.

Sheeran, P., Milne, S., Webb, T.L., \& Gollwitzer, P.M. (2005). Implementation intentions and health behaviours. In M. Conner, \& P. Norman (Eds.), Predicting health behaviour: Research and practice with social cognition models (2nd ed., pp. 276-323). Buckingham: Open University Press.

Sheeran, P., Webb, T.L., \& Gollwitzer, P.M. (2005). The interplay between goal intentions and implementation intentions. Personality and Social Psychology Bulletin, 31, 87-98.

Sniehotta, F.F., Araújo-Soares, V., \& Dombrowski, S.U. (2007). Randomised controlled trial of a one-minute intervention changing oral self-care behaviour. Journal of Dental Research, 86, 641-645.

Sniehotta, F.F., Scholz, U., \& Schwarzer, R. (2005). Bridging the intention-behaviour gap: Planning, self-efficacy and action control in the adoption and maintenance of physical exercise. Psychology and Health, 20, 143-160.

Sniehotta, F.F., Schwarzer, R., Scholz, U., \& Schüz, B. (2005). Action Planning and Coping Planning for Long-Term Lifestyle Change: Theory and Assessment. European Journal of Social Psychology, 35, 565-576.

Sutton, S. (2008). How does the Health Action Process Approach (HAPA) Bridge the intentionbehavior gap? An examination of the model's causal structure. Applied Psychology. An International Review, 57(1), 66-74.

White, K.M., Terry, D.J., \& Hogg, M.A. (1994). Safer sex behavior: The roles of attitudes, norms and control factors. Journal of Applied Social Psychology, 24, 2164-2192.

Willich, S.N., Muller-Nordhorn, J., Kulig, M., Binting, S., Gohlke, H., et al. (2001). Cardiac risk factors, medication, and recurrent clinical events after acute coronary disease: A prospective cohort study. European Heart Journal, 22, 276-279.

WMA. (2004). World Medical Association Declaration of Helsinki: Ethical principles for medical Research involving human subjects. Retrieved May 11, 2007, from http:/www.wma.net/e/ policy/pdf/17c.pdf

Ziegelmann, J.P., Luszczynska, A., Lippke, S., \& Schwarzer, R. (2007). Are goal intentions or implementation intentions better predictors of health behavior? A longitudinal study in orthopedic rehabilitation. Rehabilitation Psychology, 52, 97-102. 